\title{
Evaluation of Nasal Resistance by Rhinomanometry before and after Endoscopic Sinus Surgery
}

\author{
Shoji Kaneda ${ }^{1}$ Motoki Sekine ${ }^{1}$ Kousuke Saito ${ }^{1}$ Tomoaki Murakami ${ }^{1}$ Hikaru Yamamoto ${ }^{1}$ \\ Kenji Okami ${ }^{1}$ Hiroyuki Furuya ${ }^{2}$ Masahiro lida ${ }^{1}$
}

${ }^{1}$ Department of Otolaryngology, Tokai University, Kanagawa, Japan
${ }^{2}$ Basic Clinical Science and Public Health, Tokai University, School of
Medicine, Kanagawa, Japan
Address for correspondence Shoji Kaneda, MD, Department of Otolaryngology, Tokai University, 143 Shimokasuya, Isehara, Kanagawa, 259-1193, Japan (e-mail: bolokun2002@yahoo.co.jp).

Int J Pract Otolaryngol 2019;2:e18-e25.

\begin{abstract}
Keywords

- nasal obstruction

- nasal resistance

- rhinomanometry

- before and after ESS

With the objective of identifying the factors affecting the nasal resistance in patients undergoing sinus surgery, we evaluated the data of 95 patients with chronic sinusitis who underwent endoscopic sinus surgery (ESS) between 2015 and 2018 at our hospital. We determined the nasal resistance by rhinomanometry and also patients' sensation of nasal obstruction before surgery and 3 months after the ESS. In total, 49 bilateral and 46 unilateral ESS procedures were performed, including ESS alone, ESS + septoplasty, and ESS + septoplasty + conchotomy. In addition, the surgical cases were classified into single sinus or polysinus surgery, and groups with or without polyps. We compared the nasal resistance and degree of change in the nasal resistance among the groups. The same comparisons were also conducted separately in cases with nasal resistance levels of $>0.25 \mathrm{~Pa} / \mathrm{cm}^{3} / \mathrm{s}$. Nasal resistance improved following the ESS in both the bilateral and unilateral cases. In almost all the subgroups, the nasal resistance improved following ESS with the exception of the ESS with septoplasty group with nasal resistance levels of $>0.25 \mathrm{~Pa} / \mathrm{cm}^{3} / \mathrm{s}$. The degree of change in the nasal resistance was not significantly different between the ESS alone, ESS + septoplasty, and ESS + septoplasty + conchotomy groups. Similarly, the degree of change in the nasal resistance was also not significantly different between the single sinus and polysinus surgery or between patients with and without polyps. Furthermore, there were no differences among the above groups of patients with $>0.25 \mathrm{~Pa}$ / $\mathrm{cm}^{3} / \mathrm{s}$ either. The patients' sensation of nasal obstruction improved following the ESS. The nasal resistance measured by rhinomanometry was weakly correlated with the sensation of nasal obstruction prior to the ESS; however, the nasal resistance following the ESS and the degree of change of the nasal resistance following the ESS were not correlated with the sensation of nasal obstruction or the degree of change in the sensation of nasal obstruction. Future studies are needed for a precise clarification of the factors that affect nasal resistance.
\end{abstract}

\section{Introduction}

Rhinomanometry and acoustic rhinomanometry, which are used as objective methods to evaluate nasal airflow, have been published as such in the guideline by the Japanese Rhinologic Society Committee for the Standardization of Rhinomanometry. ${ }^{1-4}$ Several reports by the

received

December 27, 2018

accepted

April 15, 2019 committee members have been published on nasal airflow in nasal septum correction and conchotomy; however, there are few reports of sinus surgery. Therefore, we evaluated nasal airflow before and after surgery using rhinomanometry in patients who underwent endoscopic sinus surgery (ESS) and hereby report on the results of our evaluation.
Copyright $\odot 2019$ Georg Thieme Verlag License terms KG Stuttgart · New York 


\section{Material and Methods}

The study included 95 patients who underwent ESS for chronic sinusitis between February 2015 and February 2018; in these patients, nasal resistance was evaluated using rhinomanometry before surgery and at 3 months after surgery. Rhinomanometry was measured using the active anterior method with a nasal nozzle. Nasal resistance at $\Delta P=100$ Pa upon inhalation was defined as the representative value. The study comprised 55 male and 40 female patients, aged 13 to 80 years (mean: 54 years). In 49 bilateral ESS patients and 46 unilateral ESS patients, pre- and postoperative rhinomanometry data were compared between the two groups. Furthermore, patients were divided into three groups according to concurrent surgery, i.e., ESS alone group (62 patients), ESS + septoplasty (11 patients), and ESS + septoplasty + conchotomy (18 patients); pre- and postoperative nasal resistance were compared in each group, and the amounts of change in nasal resistance were compared among the groups. Similarly, patients were classified according to the number of sinuses opened: single sinus surgery group (32 sides) and polysinus surgery group (108 sides). They were also classified according to the presence or absence of polyps on the operative side: group without polyps (60 sides) and the group with polyps ( 85 sides); these groups were then compared. Furthermore, we conducted the same examination in patients with a preoperative nasal resistance of $\geq 0.25$ (ESS alone group: 25 patients; ESS + septoplasty group: 4 patients; ESS + septoplasty + conchotomy: 7 patients; single sinus surgery group: 29 sides; polysinus surgery group: 91 sides; group without polyps: 48 sides; and group with polyps: 76 sides). Moreover, the same examination was conducted in patients who underwent ESS alone classified into the group with polyps ( 45 sides) and the group without polyps (44 sides), after which the same test was conducted in patients without polyps who underwent ESS alone classified into the single sinus surgery group ( 16 sides) and polysinus surgery ( 28 sides) group. Pre- and postoperative comparisons were performed using the Wilcoxon signed-rank test, and intergroup comparisons were performed using the Mann-Whitney test, with $p$ values of $<0.05$ considered to indicate significant difference. In 58 patients who underwent bilateral ESS alone in whom the nasal symptom questionnaire was $\operatorname{completed~}^{5}$ ( - Fig. 1) at the same time that nasal resistance was confirmed, we compared pre- and postoperative nasal obstruction scores by Wilcoxon

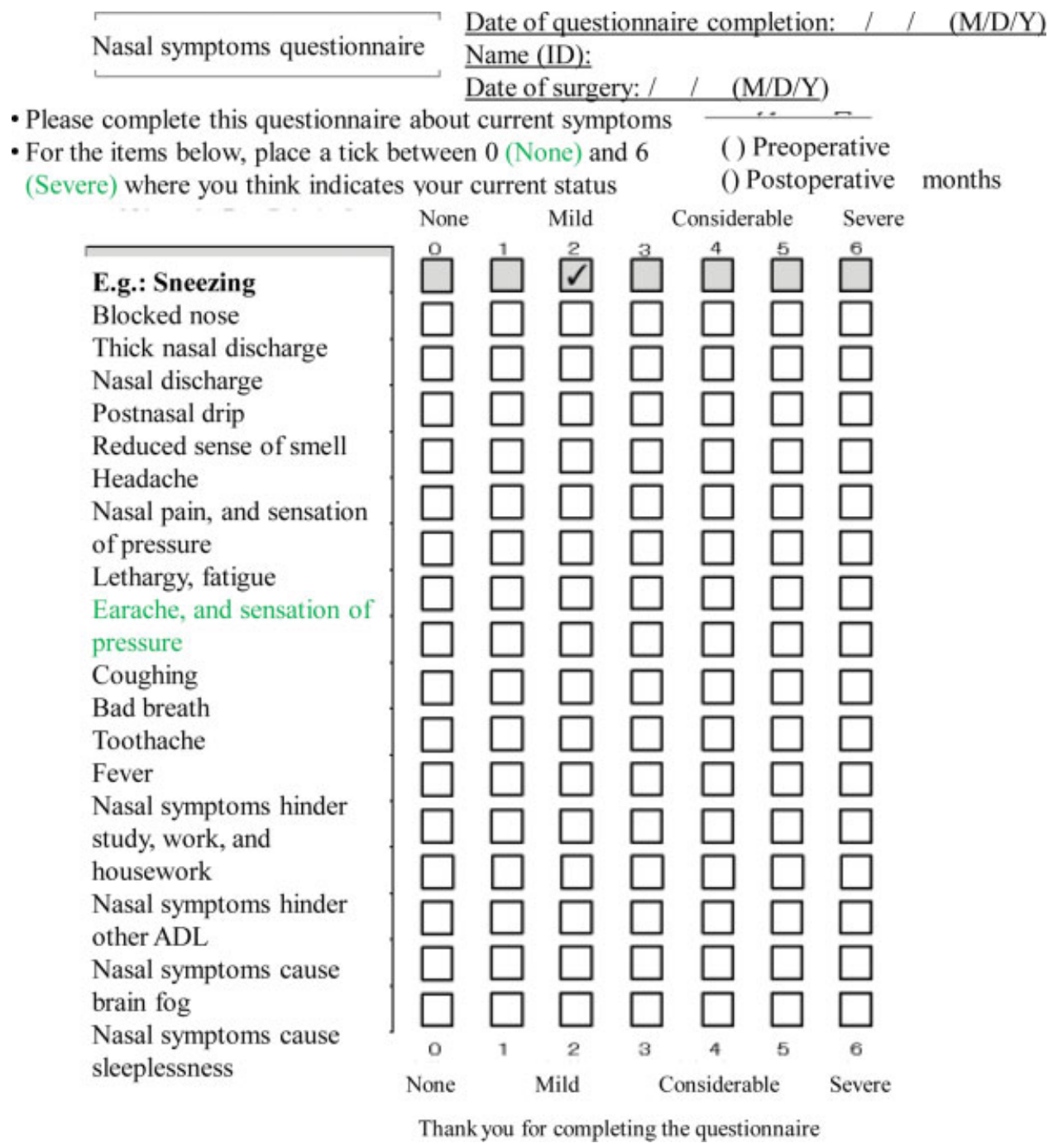

Fig. 1 Nasal symptom questionnaire. Of the questionnaire items, we investigated "nasal obstruction" in the present study. 

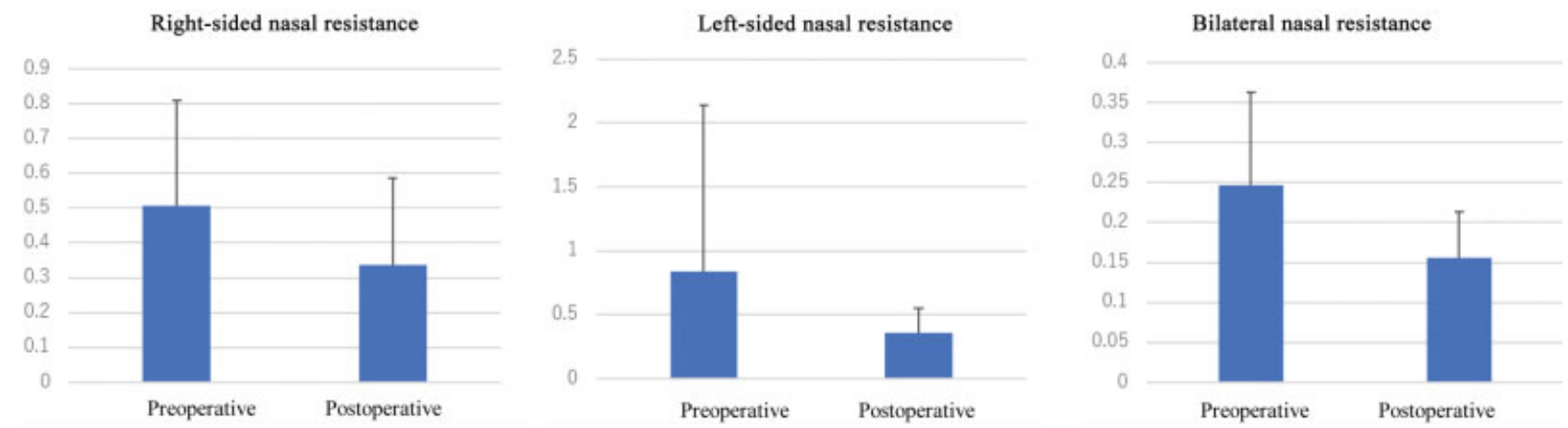

Fig. 2 Pre-and postoperative comparison in bilateral ESS patients. After surgery, the bilateral nasal resistance significantly improved. Statistical analysis was performed using the Wilcoxon signed-rank test. All $p$-values were $<0.05$. ESS, endoscopic sinus surgery.

Nasal resistance

of the affected side.

2

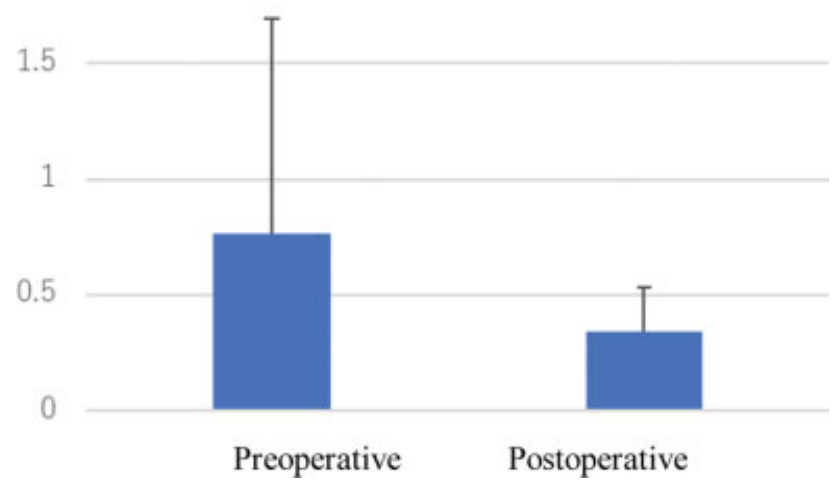

Fig. 3 Pre- and postoperative comparison in unilateral ESS patients. After surgery, the nasal resistance significantly improved. Statistical analysis was performed using the Wilcoxon signed-rank test. The $p$ value was set at $<0.05$ for all analyses. ESS, endoscopic sinus surgery. signed-rank test. We also assessed the correlation between nasal obstruction scores and nasal resistance. The present study was approved by the Institutional Review Board for Clinical Research of the Tokai University School of Medicine (14R-236).

\section{Results}

In bilateral ESS patients, the nasal resistance significantly improved in bilateral, right-sided, and left-sided procedures $(p<0.05 ;-$ Fig. 2). In unilateral ESS patients, the nasal resistance in the affected side significantly improved ( $p<0.05 ;$ - Fig. 3 ). In the groups classified by concurrent surgery, those classified by the number of sinuses opened, and those classified by the presence or absence of polyps, postoperative nasal resistance was significantly improved (-Fig. 4). In the groups similarly limited to patients with nasal resistance of $\geq 0.25$, excluding the ESS + septoplasty group ( $p=0.13$ ), the postoperative nasal resistance significantly improved $(p<0.05 ;-$ Fig. 5$)$. The intergroup comparison of the amount of change in nasal resistance revealed no significant differences between the ESS alone group and
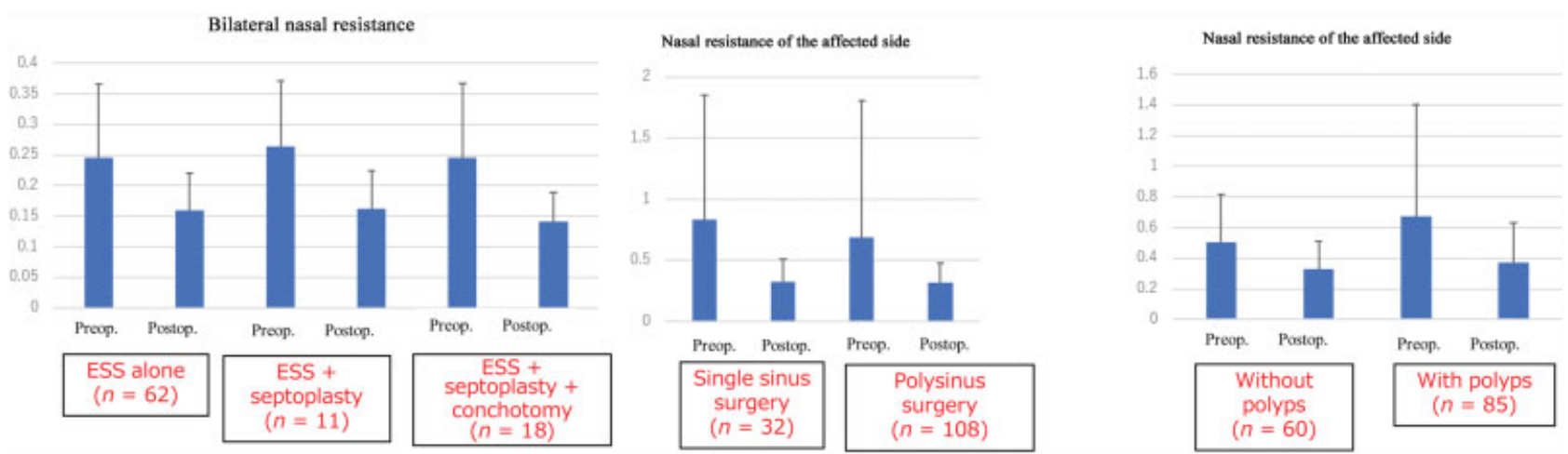

Fig. 4 Pre- and postoperative comparison of nasal airflow rate in each group, and intergroup comparison of the amount of change in the nasal resistance. Upon comparing the nasal resistance before and after surgery, we found a significant improvement in all groups including the ESS alone group, the ESS + septoplasty group, the ESS + septoplasty + conchotomy group, the single sinus surgery group, polysinus surgery group, the group without polyps, and the group with polyps. Statistical analysis was performed using the Wilcoxon signed-rank test. All p-values were $<0.05$. Upon comparing the amount of change in nasal resistance, we found no significant difference between the ESS alone group and the ESS + septoplasty group, between the ESS alone group and the ESS + septoplasty + conchotomy group, between the single sinus surgery group and polysinus surgery group, and between the group without polyps and the group with polyps. Statistical analysis was performed using the MannWhitney test. The $p$-values were $0.44,0.41,0.25$, and 0.34 , respectively. ESS, endoscopic sinus surgery. 

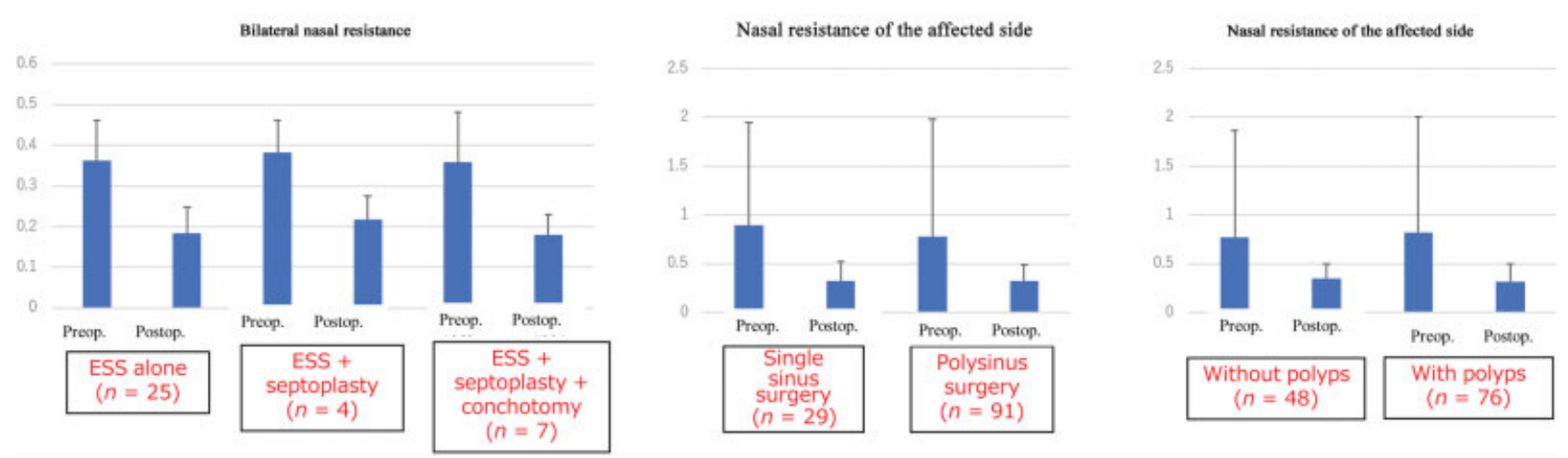

Fig. 5 Pre-and postoperative comparison of the nasal resistance in each group, and intergroup comparison of the amount of change in the nasal resistance in patients with nasal resistance of $\geq 0.25$. Upon comparing the nasal resistance before and after surgery, we found a significant improvement in all groups including the ESS alone group, the ESS + septoplasty + conchotomy group, the single sinus surgery group, polysinus surgery group, the group without polyps, and the group with polyps. No significant difference was observed in the ESS + septoplasty group. Statistical analysis was performed using the Wilcoxon signed-rank test. The $p$-value was 0.13 in the ESS + septoplasty group, and the $p$-values in the remaining groups were $<0.05$. Upon comparing the amount of change in the nasal resistance, we found no significant difference between the ESS alone group and the ESS + septoplasty group, between the ESS alone group and the ESS + septoplasty + conchotomy group, between the single sinus surgery group and polysinus surgery group, and between the group without polyps and the group with polyps. Statistical analysis was performed using the Mann-Whitney test. The $p$-values were $0.99,0.80,0.47$, and 0.30 , respectively. ESS, endoscopic sinus surgery.

ESS + septoplasty group or between the ESS and ESS + septoplasty + conchotomy group $(p=0.44$ and 0.41). The intergroup comparison of the amount of change in nasal resistance revealed no significant difference between the single sinus surgery group and polysinus surgery group $(p=0.25)$. The intergroup comparison of the amount of change in nasal resistance revealed no significant difference between the group without polyps and the group with polyps ( $p=0.34 ;$ - Fig. 4 ). When the same comparisons were made on patients after limiting them to those with nasal resistance of $\geq 0.25$, no significant differences were found $(p=0.99,0.80,0.47$, and 0.30$)$ (-Fig. 5). In the examination of the group with polyps and that without polyps among patients who underwent ESS alone, intergroup comparison of pre- and postoperative data revealed an improvement with a significant difference $(p<0.05)$; however, the intergroup comparison of the amount of change in nasal resistance revealed no significant difference ( $p=0.06$; - Fig. 6).

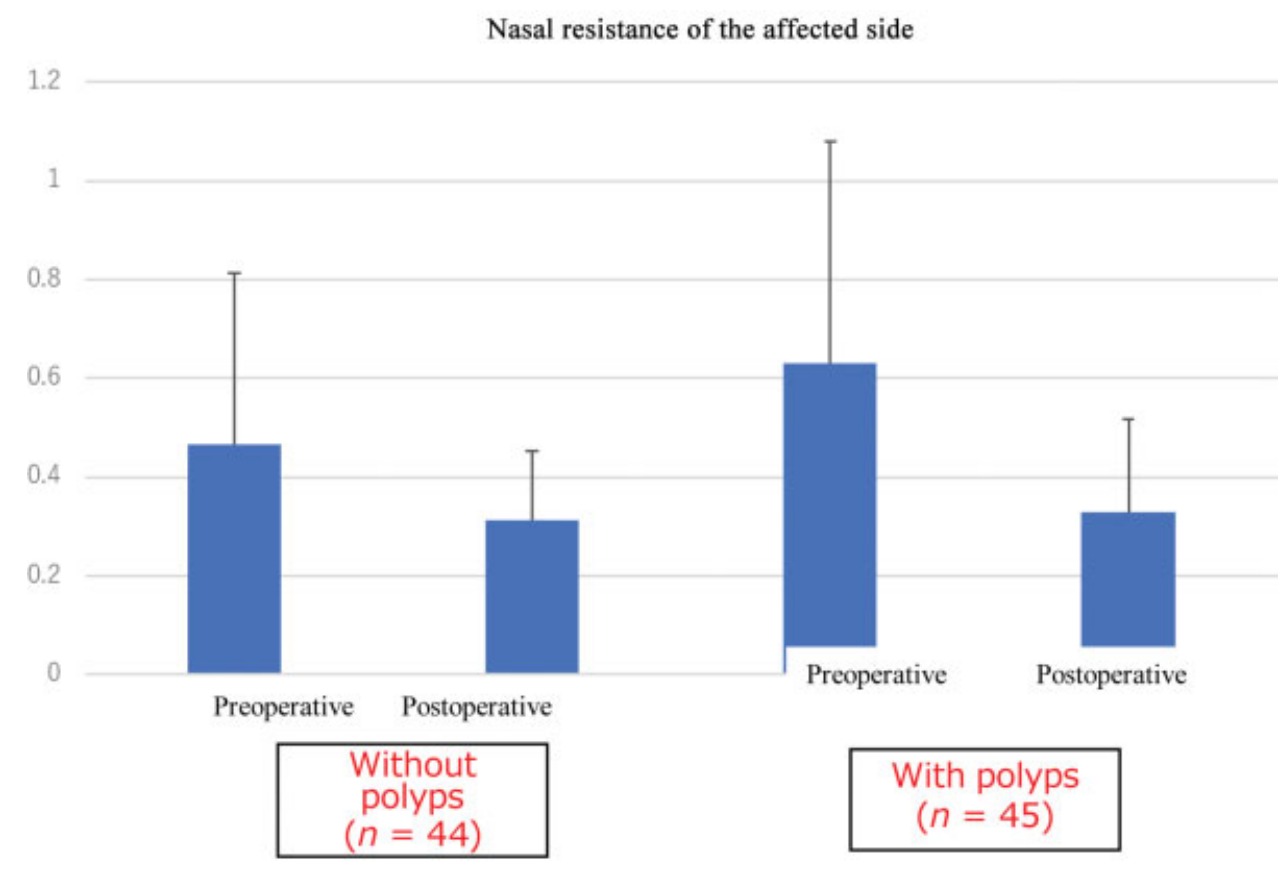

Fig. 6 Pre- and postoperative comparison of the nasal resistance in the group with polyps and the group without polyps, and intergroup comparison of the amount of change in the nasal resistance in patients who underwent ESS alone. A significant improvement was observed in postoperative nasal resistance compared with preoperatively in the group without polyps and the group with polyps. Statistical analysis was performed using the Wilcoxon signed-rank test. All $p$-values were $<0.05$. In the intergroup comparison of the amount of change in the degree of nasal resistance between the group without polyps and the group with polyps, no significant difference was observed. Statistical analysis was performed using the Mann-Whitney test, and the p-value was 0.06 . ESS, endoscopic sinus surgery. 
In the examination of the single sinus surgery group and polysinus surgery group among patients who underwent ESS alone without polyps, the comparison of pre- and postoperative nasal resistance revealed no significant difference in the single sinus surgery group ( $p=0.09$ ); however, a significant improvement was noted in the polysinus surgery group ( $p<0.05$ ). Intergroup comparison of the amount of change in the nasal resistance revealed no significant difference ( $p=0.97$; -Fig. 7 ).

The nasal obstruction scores significantly improved from a preoperative mean of 2.0 to a postoperative mean of 0.5 $(p<0.05)$, and all postoperative scores were $<2$. A weak correlation with a Spearman rank correlation coefficient of 0.3 was observed between preoperative nasal obstruction scores and resistance rate (-Fig. 8); however, the postoperative correlation coefficient of 0.1 ( - Fig. 9) indicated that there was no correlation. Between the amount of pre-topostoperative change in nasal obstruction scores and amount of pre-to-postoperative change in nasal resistance, the Spearman rank correlation coefficient was 0.203 , indicating no significant correlation (-Fig. 10).

\section{Discussion}

In the Japan Rhinologic Society, the evaluation of subjective symptoms and quality of life (QOL), evaluation using endoscopy, evaluation using computed tomography imaging, and evaluation using rhinomanometry have been proposed as a functional assessment of ESS. In the present study, before and after ESS, we evaluated the nasal resistance, which is believed to strongly correlate with nasal obstruction, i.e., the primary symptom of chronic sinusitis. Furthermore, as influencing factors, we examined the presence or absence of concurrent surgery such as septoplasty and conchotomy, the presence of any differences depending on whether single sinus surgery or polysinus surgery was performed, as well as the difference according to the presence or absence of polyps.

In the pre- and postoperative comparison, excluding the ESS + septoplasty group with nasal resistance of $\geq 0.25$, the nasal airflow rate improved with a significant difference. One group showed no significant difference; this was considered to be owing to the fact that the group had a small sample size (four patients). We believe that the reason for the improvement in the nasal resistance is direct changes resulting from surgery and indirect changes such as alleviation of paranasal sinusitis and reduction in mucosal swelling. ${ }^{6}$

According to the criteria reported by Takeuchi et al, to evaluate ESS when the preoperative nasal resistance is 100 , the postoperative nasal airflow rate is approximately $70 .{ }^{6}$ Based on this criterion, in the present study, the postoperative nasal resistance was found to be 63.4 in bilateral patients and 44.9 in the affected side of unilateral patients, indicating a sufficient improvement.

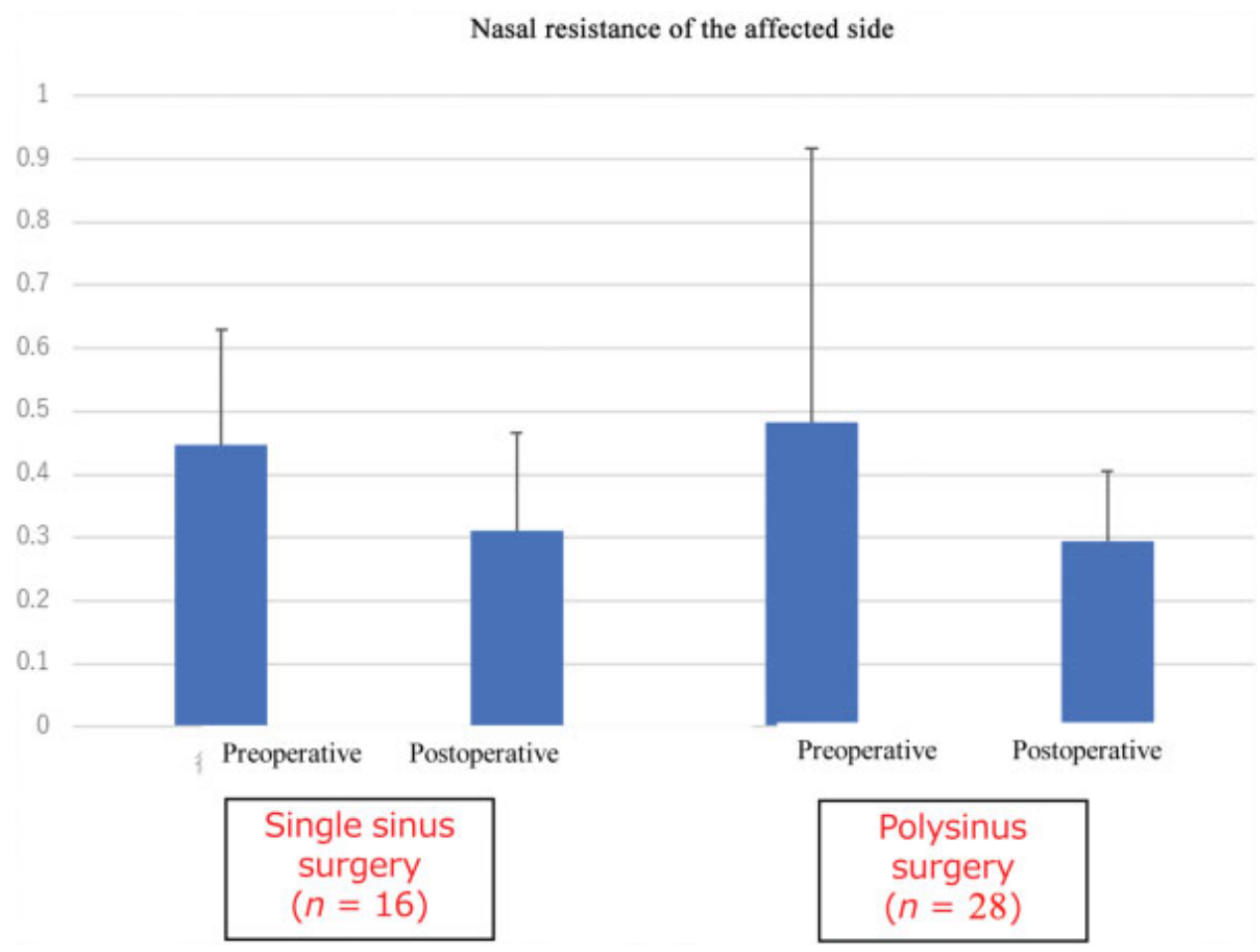

Fig. 7 Pre- and postoperative comparison of the nasal resistance in patients who underwent ESS alone without polyps in the single sinus surgery group and polysinus surgery group, and intergroup comparison of the amount of change in the nasal resistance. Upon comparing the nasal resistance before and after surgery in patients in the single sinus surgery group, there was no significant difference observed; however, the nasal resistance significantly improved in the polysinus surgery group. Statistical analysis was performed using the Wilcoxon signed-rank test. The $p$ value was 0.09 in the single sinus surgery group, and $<0.05$ in the polysinus surgery group. Upon comparing the amount of change in the nasal resistance between the single sinus surgery group and polysinus surgery group, there was no significant difference observed. Statistical analysis was performed by Mann-Whitney test, and the $p$-value was 0.97 . ESS, endoscopic sinus surgery. 


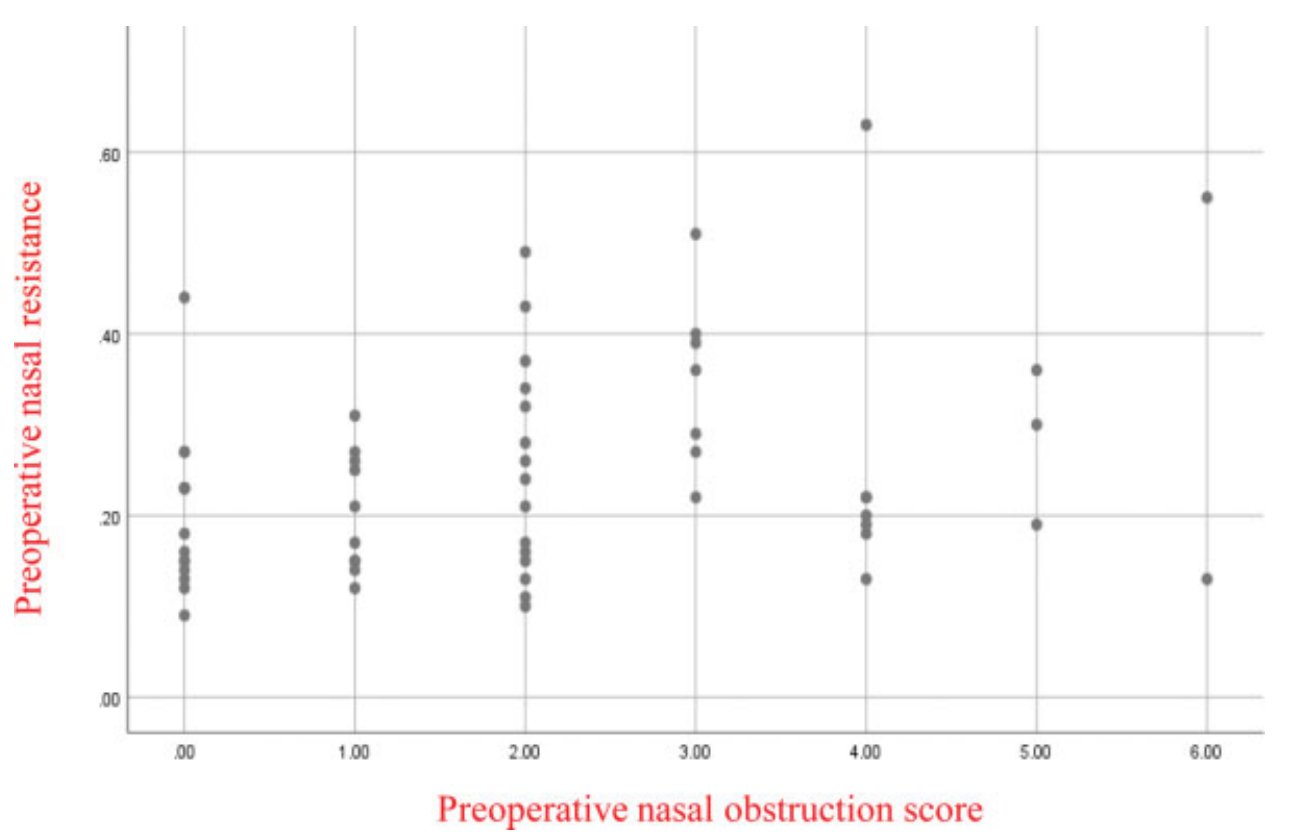

Fig. 8 Scatter diagram of the nasal resistance and nasal symptoms scores (preoperative). A weak correlation was observed with a Spearman rank correlation coefficient of 0.3 .

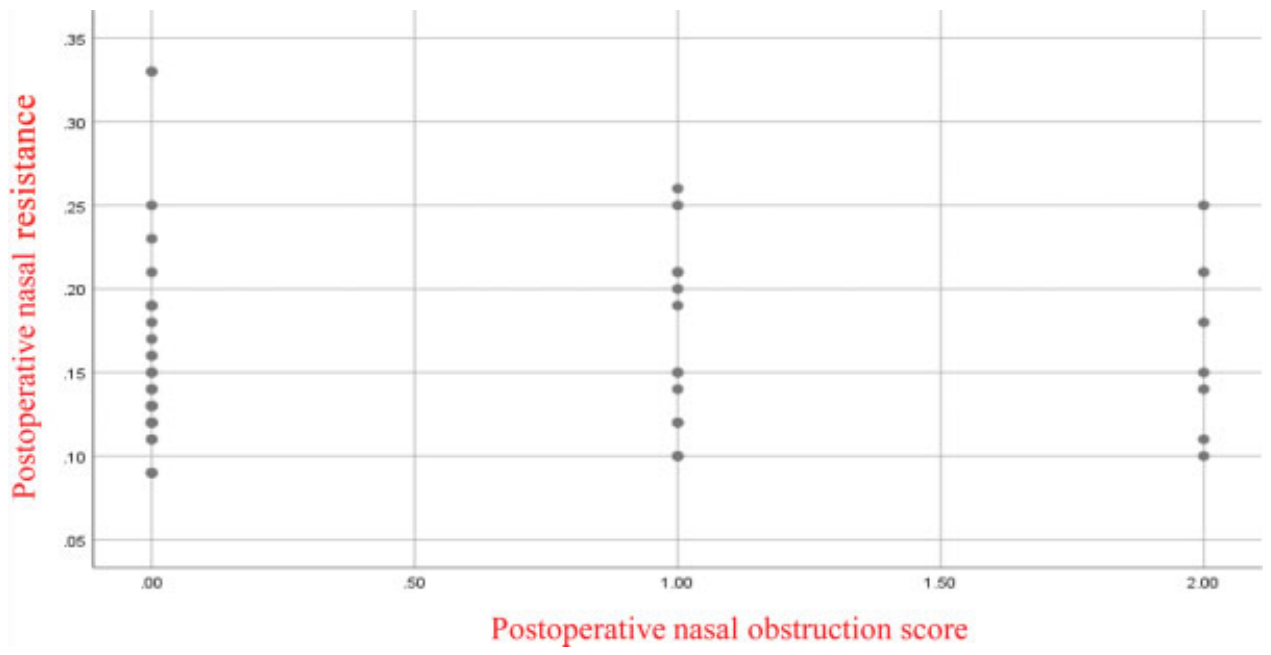

Fig. 9 Scatter diagram of the nasal resistance and nasal obstruction scores (postoperative). A significant correlation was not observed with a Spearman rank correlation coefficient of 0.1 .

Upon examining the effect of septoplasty with bilateral submucosal inferior turbinectomy on patients with sleeprelated sleeping disorders and/or snoring habit, it has been reported that the nasal resistance significantly improved after surgery. ${ }^{7,8}$ In the present study, we conducted an intergroup comparison of the amount of change in nasal resistance in patients classified according to concurrent surgery into the ESS alone group, the ESS + septoplasty group, and the ESS + septoplasty + conchotomy group. Compared with the ESS alone group, we predicted that the amount of pre-to-postoperative change in nasal resistance would be greater in the concurrent surgery groups. However, the results revealed no difference between groups in the amount of change. This could be attributed to a sufficient improvement in postoperative nasal resistance as a result of appropriate choice of surgery for each individual patient. Numminen et al compared middle meatus antrostomy and ethmoidectomy using rhinomanometry, and reported that patients who underwent ethmoidectomy showed a greater improvement in nasal resistance. ${ }^{9}$ To examine the effect of the number of sinuses opened on nasal airflow rate, we classified patients into the single sinus surgery group and polysinus surgery group; however, an intergroup comparison of the amount of change in nasal resistance revealed no significant difference. To exclude the effect of concurrent surgery and the effect of polyps, we conducted the same examination in patients limited to those who underwent ESS alone and did not have polyps; however, no significant difference was found. It is conceivable that the effect on nasal resistance differs depending on 


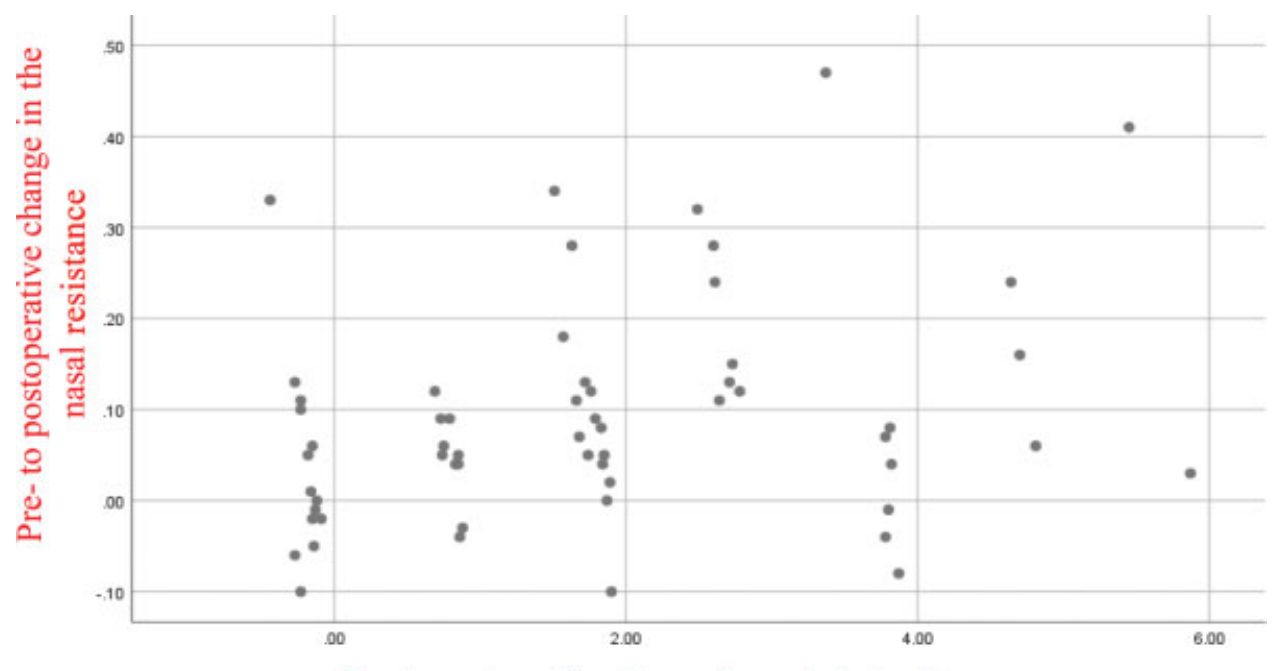

Pre- to postoperative change in nasal obstruction scores

Fig. 10 Scatter diagram of the amount of pre-to-postoperative change in the nasal resistance, and the amount of pre-to-postoperative change in nasal obstruction scores. A significant correlation was not observed with a Spearman rank correlation coefficient of 0.203.

the site of the opened sinus, which we believe could have been the reason why there was no difference found in the study limited to the number of opened sinuses alone. We also investigated the presence or absence of polyps, which is believed to greatly affect nasal resistance but found no difference between the groups. To exclude the effect of concurrent surgery, we conducted the same examination with patients limited to those who underwent ESS alone; however, there was no significant difference. The effect of polyps on nasal resistance differs depending on their size and location, which therefore could have prevented a significant difference from being found in our comparison according to the presence or absence of polyps alone. In patients with normal preoperative nasal resistance, the amount of pre-to-postoperative change is small; therefore, we conducted the same examination with patients limited to those with nasal resistance of $\geq 0.25$; however, no significant difference was found in any of the intergroup comparisons. In addition to the items examined in the present study, the nasal resistance is affected by multiple factors such as the state of the nasal mucosa, the amount of nasal discharge, and the nasal cycle. In the future, multivariate analyses need to be performed on a larger subject sample with factors classified in more detail.

Naito et al evaluated nasal airflow and nasal obstruction pre- and postoperatively using rhinomanometry and acoustic rhinomanometry in 50 patients who underwent nasal sinus and paranasal sinus surgery, and reported an improvement with a significant difference. ${ }^{10}$ At our hospital, we evaluate subjective symptoms and QOL using a questionnaire in conjunction with pre- and postoperative rhinomanometry. In the present study, nasal obstruction scores significantly improved after surgery, and all postoperative scores were $<2$. Furthermore, we examined the correlation between nasal obstruction scores and nasal airflow rates. As a result, a weak correlation was found preoperatively; however, no correlation was noted postoperatively. A weak correlation both preoperatively and postoperatively has been reported between nasal resistance and the sinonasal outcome test-20 (SNOT-20), which is an evaluation tool for subjective symptoms and QOL. ${ }^{11}$ In the present study, we only examined nasal obstruction items. In the future, correlations with subjective evaluations and nasal resistance combined with other questionnaire items need to be investigated.

\section{Conclusions}

We compared the nasal resistance before and after ESS. The nasal resistance adequately improved after surgery for most evaluation items. Intergroup comparison between the single surgery group and the concurrent surgery group revealed no difference in the amount of change in nasal resistance. Intergroup comparison between the single sinus surgery group and the polysinus surgery group as well as that between the group without polyps and the group with polyps revealed no difference in the amount of change in nasal resistance. Intergroup comparison between the group without polyps and the group with polyps in patients who underwent ESS alone revealed no difference in the amount of change in nasal resistance, and in patients without polyps who underwent ESS alone, intergroup comparison between the single sinus surgery group and polysinus surgery group also revealed no difference in the amount of change in nasal resistance. In future, the effect of other factors on nasal resistance should be examined. Furthermore, while a weak correlation was found between preoperative nasal resistance and nasal obstruction, there was no correlation observed between the postoperative nasal resistance and the amount of pre-to-postoperative change. In future studies, we intend to examine the relationship of subjective evaluations other than nasal obstruction with nasal resistance.

\section{Conflict of Interest}

None declared. 


\section{References}

1 Naito K, Miyazaki S, Nonaka S. Guideline for measuring the nasal resistance by rhinomanometry. Jap J Rhinol 2001;40:327-331

2 Kase Y, Ohki M. Guideline for acoustic rhinometry. Jap J Rhinol 2001;40:322-326

3 Takeuchi H. Evaluation of nasal resistance. Jiten 2011;54:390-397

4 de Paula Santos R, Habermann W, Hofmann T, Stammberger H. Pre and post functional endoscopic sinus surgery nasal cavity volume assessment by acoustic rhinometry. Rev Bras Otorrinolaringol 2006;72(04):549-553

5 Yoshida T, Kojima J, Mori E, et al. Changes in quality of life and subjective symptoms following endoscopic sinus surgery. Jiten 2010;53:293-299

6 Takeuchi H, Kase Y, Naito K, et al. Pre- and postoperative objective evaluations using rhinomanometry and acoustic rhinomanometry in patients undergoing paranasal sinus surgery. Jap J Rhinol 2012;51:455-461
7 Hisamatsu K, Makiyama K, Hirai R. Effect of septoplasty with bilateral submucosal inferior turbinectomy on snoring and sleepdisordered breathing. Jap J Rhinol 2009;48:349-354

8 Takahashi R, Ohbuchi T, Hohchi N, et al. Effect of septoplasty and turbinectomy on obstructive sleep apnea syndrome [in Japanese]. Nippon Jibiinkoka Gakkai Kaiho 2013;116(07): 789-792

9 Numminen J, Dastidar P, Rautiainen M. Influence of sinus surgery in rhinometric measurements. J Otolaryngol 2004;33(02):98-103

10 Naito K, Miyata S, Saito S, Sakurai K, Takeuchi K. Comparison of perceptional nasal obstruction with rhinomanometric and acoustic rhinometric assessment. Eur Arch Otorhinolaryngol 2001;258 (10):505-508

11 Prus-Ostaszewska M, Wysocki J, Niemczyk K, Balcerzak J. The correlation of the results of the survey SNOT-20 of objective studies of nasal obstruction and the geometry of the nasal cavities. Otolaryngol Pol 2017;71(02):1-7 